\title{
Ethylene spectroscopy using a quasi-room-temperature quantum cascade laser
}

\author{
Stéphane Schilt*, Luc Thévenaz, E. Courtois, Philippe A. Robert \\ Metrology Laboratory, EPFL, Swiss Federal Institute of Technology, CH-1015 Lausanne, Switzerland
}

\begin{abstract}
Spectroscopic measurements on ethylene were performed using a quasi-room-temperature quantum cascade (QC) laser operated in pulsed mode in the $10.3 \mu \mathrm{m}$ range. Using transmission spectroscopy, a broadening of the ethylene absorption spectrum was observed with increasing laser pulse duration, due to an increase of the laser linewidth. This linewidth was determined from the measured absorption spectra, showing a value of $0.04 \mathrm{~cm}^{-1}$ for a $20 \mathrm{~ns}$ pulse duration and an enhancement coefficient of $6.5 \times 10^{-3} \mathrm{~cm}^{-1}$ per ns in the 20-50 ns pulse length range. Photoacoustic (PA) detection of ethylene was also performed using the QC laser and a resonant PA cell, with a detection limit of $60 \mathrm{ppm}$. (C) 2002 Elsevier Science B.V. All rights reserved.
\end{abstract}

Keywords: Quantum cascade lasers; Infrared spectroscopy; Photoacoustic spectroscopy

\section{Introduction}

Mid-infrared $(3-20 \mu \mathrm{m})$ is an important spectral range for gas spectroscopy, as most of the atmospheric constituents and pollutants present strong rotational-vibrational absorption bands in this region [1]. The novel semiconductor quantum cascade (QC) lasers [2] have shown very promising properties for infrared gas spectroscopy and are probably the future sources for this kind of application. Based on a fundamentally new principle (intersubband transition), their wavelength can be tailored using the same material over a broad band in the mid-infrared. Unlike conventional semiconductor laser diodes, their emission

\footnotetext{
* Corresponding author

E-mail address: stephane.schilt@epfl.ch (S. Schilt).
}

wavelength does not depend on the band gap of the material, but is entirely determined by the quantum confinement of the electrons in a quantum structure grown by Molecular Beam Epitaxy. This allows to use conventional and technologically-matured III-V semiconductor materials (GaAs, InP) to design and fabricate mid-infrared lasers in a spectral range where narrower band gap materials are usually used ( $\mathrm{PbS}, \mathrm{PbSe})$. In addition to the easier-to-process materials used for their manufacturing, these unipolar devices present several advantages in comparison with conventional lead-salt lasers previously used for mid-infrared spectroscopy. First, lead-salt lasers require a cryogenic cooling [3], that is poorly acceptable for site applications. QC lasers, on the other hand, are thermo-electrically cooled or may be operated at room temperature [4,5], only in 
pulsed mode so far. But continuous wave $(\mathrm{CW})$ operation at room temperature is expected in near future. Secondly, QC lasers show a much higher output power than lead-salt lasers, thanks to the unique cascading scheme used in these unipolar devices: after the emission of a photon, an electron is still in the conduction band and can be recycled to emit other photons in adjacent active regions of the laser. This device is thus made of a large number of stages (25-30 typically) and many photons per electron are emitted, enabling high optical powers (tens to hundreds of $\mathrm{mW}$ ) compared with lead-salt lasers (only several hundreds of $\mu \mathrm{W}$ ). Finally, lead-salt lasers are available only as Fabry-Perot cavities and generally present a strongly multimode spectrum resulting in a poor spectral purity. QC lasers are quasi single-mode in $\mathrm{CW}$ operation, even for FabryPerot cavity [4]. Although they become strongly multimode when pulsed, single-mode emission can be granted using a distributed feedback (DFB) structure [6]. In that case, single-mode emission has been demonstrated in a temperature range from 80 to $390 \mathrm{~K}[6,7]$.

In this paper, we report spectroscopic measurements on ethylene $\left(\mathrm{C}_{2} \mathrm{H}_{4}\right)$ using a quasi-roomtemperature DFB-QC laser emitting in the 10.3 $\mu \mathrm{m}$ range. Two different methods were experimented. Transmission spectroscopy with direct detection using an infrared detector was used for spectra analysis. The effect of the laser pulse duration on the measured $\mathrm{C}_{2} \mathrm{H}_{4}$ absorption spectrum was analysed. A broadening of the spectrum was observed, showing an increase of the laser linewidth with pulse duration. The linewidth enhancement was determined as a function of the pulse duration from the apparent profile of an absorption line of ethylene. Photoacoustic spectroscopy was used for low concentrations measurements. This technique was performed by coupling the QC laser emission into a resonant photoacoustic (PA) cell.

\section{Characterisation of the QC laser emission}

A DFB-QC laser emitting in the $10.3 \mu \mathrm{m}$ range (Alpes Lasers) was mounted in a hermetic home- made housing. It was cooled using a 2-stage Peltier element and a temperature regulator. The laser beam exits the housing through a $25 \mathrm{~mm}$ $\mathrm{ZnSe}$ window. The laser was operated in pulsed mode. The current pulses were provided by a pulser with a pulse duration varying between 20 and $50 \mathrm{~ns}$ and a duty cycle of $1-2 \%$.

The wavelength of a QC laser can be tuned by changing either the laser temperature (for coarse tuning) or the laser current (for fine tuning). The laser spectrum was analysed using a $75 \mathrm{~g} \mathrm{~mm}^{-1}$ grating monochromator and a thermo-electrically cooled $\mathrm{HgCdTe}$ detector (Vigo). Fig. 1 shows the laser spectra obtained at different temperatures. The width of the laser lines shown in this figure is limited by the monochromator resolution and does not represent the real laser linewidth. A tuning range of more than $4.5 \mathrm{~cm}^{-1}$ and centred at $969.5 \mathrm{~cm}^{-1}$ was achieved by changing the laser temperature over $50{ }^{\circ} \mathrm{C}$. From these spectra, a temperature tuning coefficient of $-0.095 \mathrm{~cm}^{-1}$ per ${ }^{\circ} \mathrm{C}$ was obtained. In the same way, a current tuning coefficient of $-0.19 \mathrm{~cm}^{-1} \mathrm{~A}^{-1}$ was obtained as a function of the laser current.

The spatial distribution of the laser emission was measured using a small surface $(250 \times 250$ $\mu \mathrm{m}) \mathrm{HgCdTe}$ detector mounted on a motorised translation stage directly at the output of the laser housing, i.e. with no collecting optics. The detected power was measured in both directions as a function of the detector position, that gave the

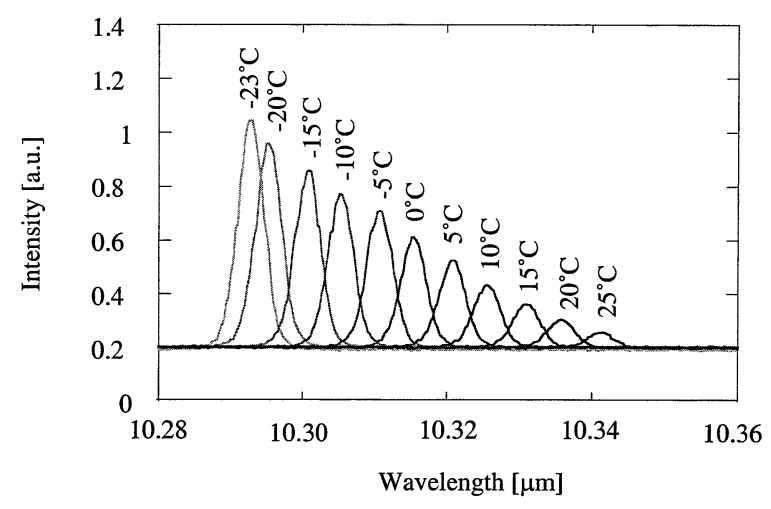

Fig. 1. Laser spectra measured at different temperatures using a grating monochromator. The laser current is $5.2 \mathrm{~A}$, the pulse duration $30 \mathrm{~ns}$ and the repetition frequency $700 \mathrm{kHz}$. 


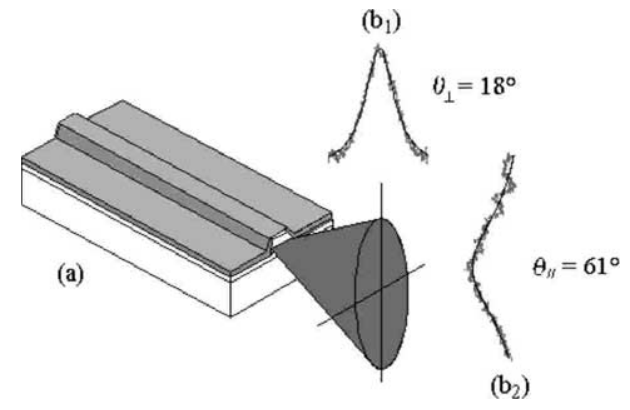

Fig. 2. Divergence of the QC laser emission. (a) Schematic representation. (b) Spatial distribution of the laser beam measured in the horizontal $\left(b_{1}\right)$ and vertical $\left(b_{2}\right)$ directions. The grey points are experimental data and the black lines are the results of a fit.

horizontal and vertical profiles of the laser beam. The measured profiles were then fitted to a Lorentzian curve, that turns out to give a better fit than a Gaussian curve [8]. The divergence angle of the laser emission was determined by measuring the beam diameter (full width at half maximum, FWHM) at different distances from the laser. Divergence of $18^{\circ}$ horizontally and $61^{\circ}$ vertically was obtained. Fig. 2 illustrates an example of the beam distributions measured in both directions. The laser divergence is also shown schematically. It appears to be much higher in the vertical direction than in the horizontal, in opposition to previous results obtained on Fabry-Perot QC lasers emitting at the same wavelength [8]. Due to this high divergence, the laser emission is poorly collected by a collimating optics, resulting in large optical losses.

\section{Transmission spectroscopy}

Fig. 3 shows the experimental arrangement used for transmission spectroscopy. The laser emission is collected and collimated with two AR coated $\mathrm{ZnSe}$ lenses (10 and $50.6 \mathrm{~mm}$ focal lengths)

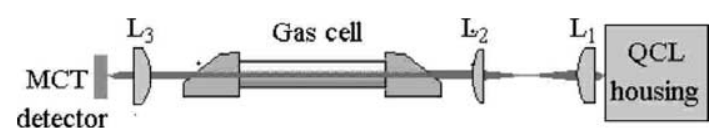

Fig. 3. Experimental set-up for transmission spectroscopy. (a)

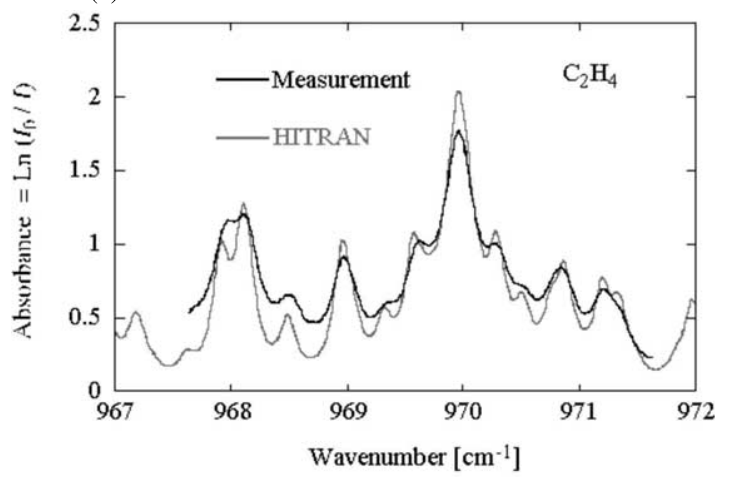

(b)

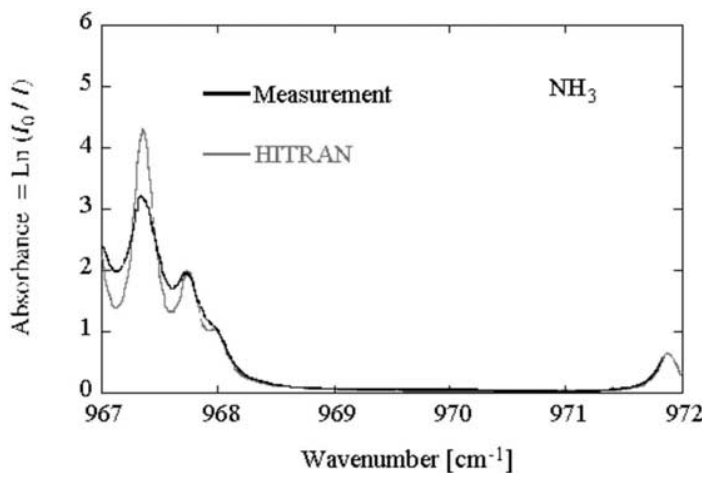

Fig. 4. Absorption spectra of $1.1 \%$ ethylene (a) and $0.24 \%$ ammonia (b) measured in a $31.5 \mathrm{~cm}$ optical pathlength in the spectral range of the QC laser. Black lines are experimental data and grey lines are taken from the HITRAN database. The laser was driven by $30 \mathrm{~ns}$ pulses at a repetition frequency of $700 \mathrm{kHz}$.

to properly size the beam. It passes through a 31.5 $\mathrm{cm}$ gas cell with $\mathrm{ZnSe}$ Brewster-angled windows. The beam is then focused on a thermo-electrically cooled $\mathrm{HgCdTe}$ detector.

Several absorption lines of ethylene and ammonia are observed within the emission range of the laser. Fig. 4 shows the absorption spectra measured when the cell was filled, respectively, with $1.1 \% \mathrm{C}_{2} \mathrm{H}_{4}$ and $0.24 \% \mathrm{NH}_{3}$ in air. These spectra were obtained by tuning the laser temperature between -20 and $25{ }^{\circ} \mathrm{C}$. The experimental results show a good agreement with the HITRAN database [9] in the position of the lines, but not in their magnitude. The measured lines are smaller and broader than the lines given by HITRAN. For example, the two $\mathrm{C}_{2} \mathrm{H}_{4}$ lines around $968 \mathrm{~cm}^{-1}$ are 
not resolved experimentally. This effect is due to the laser linewidth, which is not negligible in comparison to the width of the absorption lines. The broad width of pulsed DFB-QC lasers is thought to be produced by a chirp of the laser wavelength during the pulse, due to a thermal heating of the laser [6]. For this reason, it is preferable to drive the laser with very short current pulses. The effect of the pulse duration on the strong absorption line of $\mathrm{C}_{2} \mathrm{H}_{4}$ at $969.96 \mathrm{~cm}^{-1}$ has been analysed. This line has a broad width $\left(0.24 \mathrm{~cm}^{-1}\right.$ at atmospheric pressure $)$ as it consists of several overlapping lines of $\mathrm{C}_{2} \mathrm{H}_{4}$. The absorption spectrum was recorded for different pulse lengths, ranging between 20 and $50 \mathrm{~ns}$, as illustrated in Fig. 5. These spectra result from the convolution of the true $\mathrm{C}_{2} \mathrm{H}_{4}$ absorption spectrum with the laser linewidth. This linewidth can thus be determined from the measured spectra and the true spectrum given by HITRAN and shown in Fig.
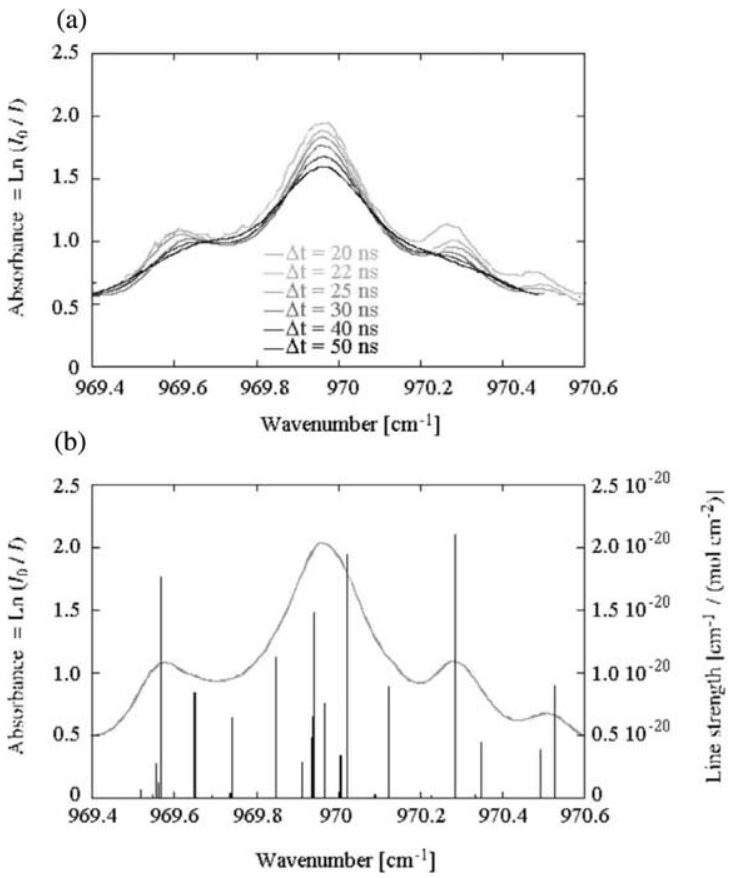

Fig. 5. (a) Part of the ethylene absorption spectrum measured with different laser pulses lengths ranging between 20 and 50 ns. The $\mathrm{C}_{2} \mathrm{H}_{4}$ concentration is $1.1 \%$ and the optical pathlength $31.5 \mathrm{~cm}$. (b) Corresponding spectrum at atmospheric pressure given by HITRAN (in grey). The individual lines are also represented (in black) with their respective strength.

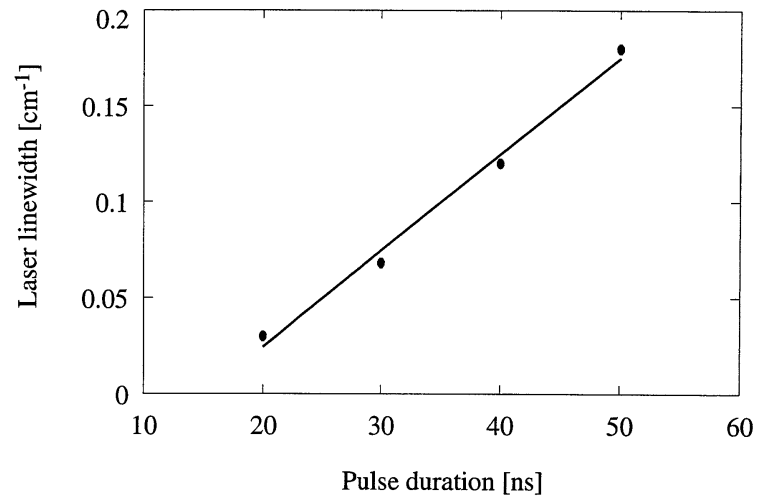

Fig. 6. Laser linewidth (FWHM) as a function of the pulse duration.

5(b). Using a deconvolution algorithm on the transmission spectra, the extracted linewidths (FWHM) are represented in Fig. 6 as a function of the pulse duration. A Gaussian lineshape was considered for the laser profile, as the exact distribution was unknown. For a $20 \mathrm{~ns}$ pulse length, the linewidth is $0.04 \mathrm{~cm}^{-1}$ and then it broadens by $6.5 \times 10^{-3} \mathrm{~cm}^{-1} \mathrm{~ns}^{-1}$ in the $20-50 \mathrm{~ns}$ pulse length range. These results agree with the value published by Zahniser [10], who measured a Gaussian FWHM of $0.012 \mathrm{~cm}^{-1}$ using a similar QC laser with a 14 ns pulse duration. Higher linewidths have been obtained by other investigators (Sonnenfroh [11], Namjou [12]), but with different QC lasers. The broadened linewidth is a penalty related to pulsed QC lasers and currently limits their use in high resolution spectroscopy.

\section{Photoacoustic spectroscopy}

PA spectroscopy is a calorimetric technique based on the PA effect, in which an acoustic wave results from the absorption of light by a species of interest. This acoustic wave (the PA signal) is directly proportional to the energy absorbed in the sample, thus to the species concentration. It can be detected using a sensitive microphone. As the PA signal is also proportional to the laser power, PA spectroscopy was mainly used with high power gas lasers in the infrared, such as $\mathrm{CO}$ or $\mathrm{CO}_{2}$ lasers [13]. Although very good perfor- 
mances have been reached with these lasers, they suffer from several drawbacks, such as their large size, limited lifetime and their restriction to discrete available wavelengths. As a result, only fortuitous coincidence between the laser line and an absorption profile can be used. On the other hand, QC lasers offer attractive properties for PA spectroscopy. In addition to the usual advantages of semiconductor devices, such as compact size and reliability, they offer continuous tunability, which allows to exactly centre the laser emission on an absorption line of the species to analyse or to scan the laser wavelength through an absorption line.

PA spectroscopy of $\mathrm{C}_{2} \mathrm{H}_{4}$ using a DFB-QC laser operating near room-temperature is presented here. The laser is operated in the same way as previously described. Fig. 7 illustrates the experimental arrangement used for PA spectroscopy. The laser emission is collimated and passes through the PA cell through $\mathrm{ZnSe}$ windows. The cell, described in [14], is made of a central cylinder of length $12 \mathrm{~cm}$ connected with two outer cylindrical buffer volumes used as notch acoustic filters. The system is operating in the first radial mode of the central cylinder, which exhibits a resonance frequency of $f \cong 10.6 \mathrm{kHz}$. Fig. 8 shows an experimental measurement of this resonance, showing a quality factor $Q$ better than 650 . The laser is pulsed at a repetition frequency of $700 \mathrm{kHz}$ and is additionally intensity-modulated at the $10.6 \mathrm{kHz}$ resonance frequency. This modulation is performed directly on the laser pulser, by gating the electrical pulses. A microphone is located at the position of the maximum acoustic amplitude in order to measure the PA signal. The microphone electrical signal is detected by a lock-in amplifier. After the cell, the laser beam is detected using a $\mathrm{HgCdTe}$ detector and a lock-in amplifier and the PA signal is normalised by the optical intensity.

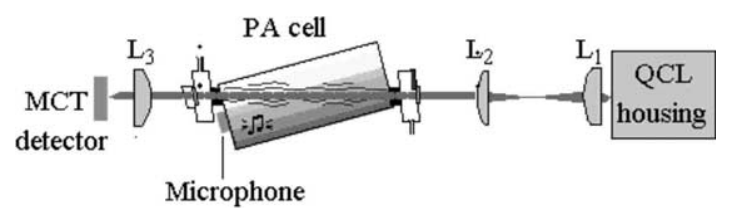

Fig. 7. Experimental set-up for PA spectroscopy.

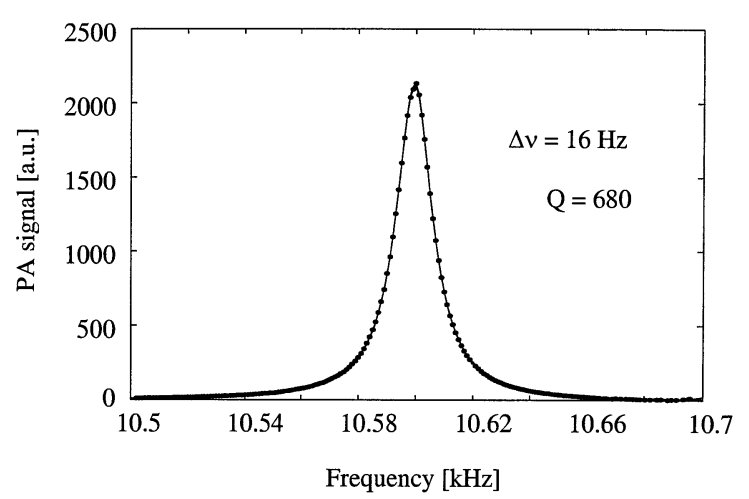

Fig. 8. Radial acoustic resonance of the PA cell, centred around $10.6 \mathrm{kHz}$. Black points are experimental data and the line is a fit by a Lorentzian function. The resonance has a quality factor $Q=680$ and a linewidth $\Delta v=16 \mathrm{~Hz}$.

The laser, operated at $T=-5.2{ }^{\circ} \mathrm{C}$ and $I=6$ A, emitted at $969.96 \mathrm{~cm}^{-1}$, which corresponds to a strong absorption line of ethylene. The PA signal was measured for different $\mathrm{C}_{2} \mathrm{H}_{4}$ concentrations in air. Fig. 9 shows the detected PA signal as a function of the $\mathrm{C}_{2} \mathrm{H}_{4}$ concentration. The microphone noise in absence of ethylene was about 0.2 $\mathrm{mV}$. This high value is mainly due to electrical noise produced by the laser driving electronics. High current pulses (6-7 A) are required for the laser operation and electromagnetic noise is radiated by the laser pulser and its low impedance copper line. This noise is detected by the microphone and the $\mathrm{HgCdTe}$ detector. Attention has been paid to reduce the noise, for example by

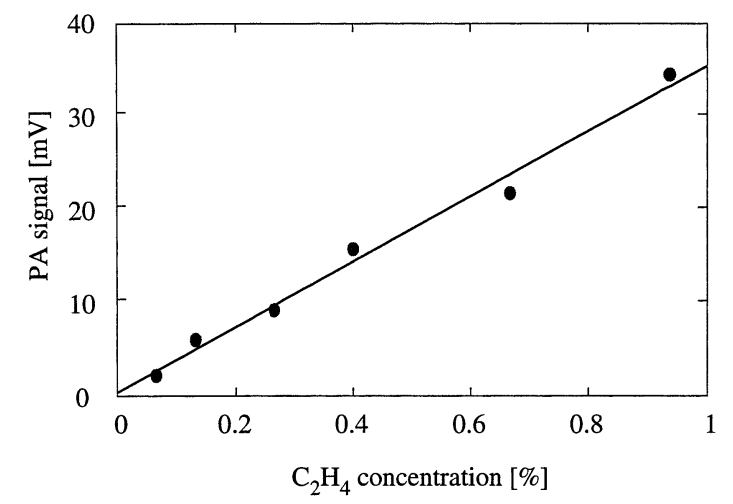

Fig. 9. Detected PA signal as a function of the ethylene concentration. 
placing the pulser in a shielded housing and by properly wiring the elements, but a high value is still present. From this noise level, a detection limit of $60 \mathrm{ppm} \mathrm{C}_{2} \mathrm{H}_{4}$ was determined. The system performances are below expectation for the moment, as a result of this high noise level, but also to the weak optical power launched into the PA cell. Indeed, the coupling of the optical power into the PA cell shows a low efficiency, due to the highly divergent emission of this QC laser. New configurations are currently investigated in order to take advantage of the specificity of the QC laser emission and to increase the light coupling into the cell. This improvement, together with a more efficient reduction of the pick-up noise, should allow to increase appreciably the system performances.

\section{Conclusion}

A DFB-QC laser has been operated in pulsed mode near room temperature for $\mathrm{C}_{2} \mathrm{H}_{4}$ spectroscopy at $10.3 \mu \mathrm{m}$. A characterisation of the laser has shown a good temperature tunability of $-0.095 \mathrm{~cm}^{-1}$ per ${ }^{\circ} \mathrm{C}$, but a strongly diverging and asymmetric emission. A full divergence angle (at half maximum) of $61^{\circ}$ was measured in the vertical direction, but only $18^{\circ}$ in the horizontal direction. This makes the collecting of the laser emission by a collimating optics poorly efficient, so that large optical losses are observed.

The absorption spectrum of ethylene measured by transmission spectroscopy has shown an important broadening of the lines, due to a laser thermal heating during the pulses, which produces a wavelength chirp. The laser linewidth has been determined from the measured apparent profile of a strong ethylene line and taken into account the true spectrum given by HITRAN. In the 20-50 ns pulse range, the linewidth enhancement factor was $6.5 \times 10^{-3}$ $\mathrm{cm}^{-1} \mathrm{~ns}^{-1}$. This high value requires the use of very short electrical pulses to drive the laser for high resolution spectroscopy.

Finally, PA spectroscopy was performed using the QC laser with a resonant PA cell. A detec- tion limit of $60 \mathrm{ppm} \mathrm{C}_{2} \mathrm{H}_{4}$ was achieved with the current performances of the system. Improvement of these performances are expected both by increasing the optical power launched in the PA cell and by reducing the noise level by a proper isolation of the laser pulser from the microphone.

\section{Acknowledgements}

The authors are grateful to Jérôme Faist, Thierry Aellen and Matthias Beck, from the University of Neuchâtel, Switzerland, for manufacturing the QC laser and fruitful discussions. E. Courtois is on leave from Ecole Centrale de Lyon, France.

\section{References}

[1] D.S. Bomse, D.C. Hovde, D.B. Oh, J.A. Silver, A.C. Stanton, Diode laser spectroscopy for on-line chemical analysis, SPIE Proc. 1681, Optically based methods for process analysis, pp. 138-148, 1992.

[2] J. Faist, F. Capasso, D.L. Sivco, C. Sirtori, A.L. Hutchinson, A.Y. Cho, Science 264 (1994) 553-556.

[3] M. Tacke, Infrared Phys. Technol. 36 (1995) 447-463.

[4] C. Sirtori, J. Faist, F. Capasso, D.L. Sivco, A.L. Hutchinson, A.Y. Cho, IEEE Photon. Technol. Lett. 9 (1997) 294-296.

[5] F. Capasso, J. Faist, C. Sirtori, A.Y. Cho, Solid State Commun. 102 (2-3) (1997) 231-236.

[6] J. Faist, C. Gmachl, F. Capasso, C. Sirtori, D.L. Sivco, J.N. Baillargeon, A.Y. Cho, Appl. Phys. Lett. 70 (20) (1997) 2670-2672.

[7] D. Hofstetter, M. Beck, T. Aellen, J. Faist, Appl. Phys. Lett. 78 (4) (2001) 396-398.

[8] S. Schilt, L. Thévenaz, P.A. Robert, Characterisation of the emission properties of a quantum cascade laser for spectroscopic applications, submitted in IEEE J. Quantum. Electr.

[9] L.S. Rothman, et al., J. Quant. Spectrosc. Radiat. Transfer 60 (1998) 665-710.

[10] M.S. Zahniser, Atmospheric trace gas measurements with pulsed-quantum cascade lasers: sub-ppb ammonia detection, Quantum Cascade Laser Workshop, Freiburg, 22 February 2001

[11] D.M. Sonnenfroh, W.T. Rawlins, M.G. Allen, C. Gmachl, F. Capasso, A.L. Hutchinson, D.L. Sivco, J.N. Baillargeon, A.Y. Cho, Appl. Optics 40 (6) (2001) 812820.

[12] K. Namjou, S. Cai, E.A. Whittaker, J. Faist, C. Gmachl, 
F. Capasso, D.L. Sivco, A.Y. Cho, Optics Lett. 23 (3) (1998) 219-221.

[13] P.L. Meyer, M.W. Sigrist, Rev. Sci. Instr. 61 (7) (1990) 1779-1807.
[14] S. Schilt, L. Thévenaz, P.A. Robert, Trace gas measurement using IR laser diodes, in Proc. International Conference on Trends in Optical Nondestructive Testing, 3-5 May 2000, Lugano, pp. 513-518. 